\title{
New miRNA expression abnormalities in laryngeal squamous cell carcinoma
}

\author{
Magdalena Cybula ${ }^{\mathrm{a}, *}$, Łukasz Wieteska $^{\mathrm{a}}$, Magdalena Józefowicz-Korczyńska ${ }^{\mathrm{b}}$, \\ Michał Seweryn Karbownik ${ }^{\mathrm{c}}$, Weronika Lucas Grzelczyk ${ }^{\mathrm{b}}$ and Janusz Szemraj ${ }^{\mathrm{a}}$ \\ ${ }^{a}$ Department of Medical Biochemistry, Medical University of Lodz, Lodz, Poland \\ ${ }^{\mathrm{b}}$ Department of Otolaryngology and Oncological Laryngology, Medical University of Lodz, Lodz, Poland \\ ${ }^{\mathrm{c}}$ Department of Pharmacology and Toxicology, Medical University of Lodz, Lodz, Poland
}

\begin{abstract}
.
BACKGROUND: Although the development of novel diagnostic and treatment strategies concerning laryngeal cancer is highly intensive, the survival rate remains virtually unchanged. Small non-coding RNAs appear to be very promising biomarkers - and so remain the focus of extensive investigation in laryngeal cancer.

OBJECTIVE: We examined the expression of five miRNA and five genes related to cancer whether they could be potential laryngeal cancer biomarkers.

METHODS: We performed an analysis in 47 patients diagnosed with laryngeal cancer. The qPCR technique was used to investigate the expression profile.

RESULTS: While miR-21-3p and miR-525-5p were found to be significantly up-regulated, miR-139-3p and miR-885-5p expression is lower in laryngeal cancer. Moreover, PIK3R1 and HACE1 were found to be also down-regulated.

CONCLUSIONS: The change in miRNA expression is frequent than the expression of other tested genes. The expression of passenger strands such as miR-21-3p and miR-139-3p, which are rarely investigated, is also significantly affected in laryngeal cancer. While PIK3R1, HACE1, miR-139-3p, and miR-885-5p may act as tumor suppressor genes in the studied tumour type, miR-21-3p and miR-525-5p seem to have oncogenic properties. Our findings suggest that miR-885-5p and PIK3RI are the best indicators for the classification of laryngeal cancer tissue and normal mucosa.
\end{abstract}

Keywords: Laryngeal squamous cell carcinoma, miRNA, expression

\section{Introduction}

Head and neck cancers constitute a diverse group of malignancies with laryngeal cancer being one of the most frequently encountered. Furthermore, it is the $6^{\text {th }}$ most common cancer worldwide [23]. Between $95 \%$ and $98 \%$ of these tumours have a squamous origin. Laryngeal squamous cell carcinoma (LSCC) development, like other epithelial malignancies, is a multistep process caused by accumulation of molecular

* Corresponding author: Magdalena Cybula, Department of Medical Biochemistry, Medical University of Lodz, Mazowiecka Street 6/8, 92-215 Lodz, Poland. Tel.: +48 4227256 88; Fax: +48 42272 56 79; E-mail: magdalenaa.cybula@gmail.com. abnormalities in the epithelium [30]. As far as carcinogenesis in LCSS is concerned, there are well established risk factors like tobacco smoking and alcohol consumption. In addition, human papillomavirus (HPV) infections have been suggested to play a significant role in the progression of laryngeal cancer $[16,40]$. There is some evidence that positive family history of laryngeal cancer or other HNSCC (head and neck squamous cell carcinoma) may increase the risk of LCSS development [3,32]. The male/female ratio for the incidence of LCSS is significantly higher than in other tumours in this group [32]. Stages I and II (early) of laryngeal squamous cell carcinoma are treated by surgery and/or radiotherapy. While more advanced disease (stage III and IV) is usually treated by surgery or 
chemoradiotherapy. Nowadays, the aim of therapeutic strategies is not only to preserve the larynx itself, but also, more importantly, its function [15].

miRNAs are non-protein coding, single-stranded RNAs 18-25 nucleotides in length. These genes are located within introns or exons of protein-coding genes and in intergenic regions. In addition, miRNAs have been found in many areas of the genome which are associated with cancer development: fragile sites, regions of amplification and $\mathrm{LOH}$ regions [11,36,42].

In spite of the fact that the development of novel diagnostic and treatment strategies is highly intensive, most patients are still diagnosed at advanced tumour stages, resulting in decreased survival rates. Therefore, there is a need to investigate new biomarkers for early detection of disease, as well as new genes which could act as targets in modern therapeutic approaches [27].

miRNAs are capable of negatively regulating gene expression. These small RNA molecules act by binding to the 3' untranslated region (UTR) of the target mRNA and inhibit its translation. Target recognition enables 5'end of the miRNA - specific region called the "seed region". It is estimated that miRNAs regulate almost half the total number of human genes [11,42].

The purpose of this study was to investigate the expression profile of five chosen miRNA (miR-21-3p, miR-139-3p, miR-377-5p, miR-525-5p and miR-885$5 \mathrm{p})$ based on the microarray experiment data and literature, as well as five genes: PIK3R1, CXCR4, FZD4, $H A C E 1$ and $C R K$ related to cancer which might be their potential targets. There are no information concerning the role of miR-377-5p, miR-525-5p and miR$885-5 p$ in laryngeal cancer. Furthermore, miR-21-3p and $\mathrm{miR}-139-3 \mathrm{p}$ are poorly investigated in this type of malignancy. Therefore, our aim was to examine the expression of abovementioned miRNA and genes, as well as to reveal their role in laryngeal squamous cell carcinoma.

\section{Materials and methods}

\subsection{Tissue samples}

Paired tissue samples of tumour and adjacent nonmalignant mucosa were obtained from 47 patients who underwent total or partial laryngectomy in Department of Otolaryngology and Oncological Laryngology Medical University of Lodz (Poland). After surgery samples were preserved and stored at $-80^{\circ} \mathrm{C}$. Nontumor tissue was used as the control. All subjects gave
Table 1

Clinicopathological characteristic in laryngeal cancer patients

\begin{tabular}{|c|c|}
\hline Characteristics & $\mathrm{n}(\%)$ \\
\hline \multicolumn{2}{|l|}{ Sex } \\
\hline Male & $40(85.1)$ \\
\hline Female & $7(14.9)$ \\
\hline \multicolumn{2}{|l|}{ Age (years) } \\
\hline$\geqslant 65$ & $21(44.7)$ \\
\hline$<65$ & $26(55.3)$ \\
\hline \multicolumn{2}{|l|}{$\mathrm{T}$ classification } \\
\hline $\mathrm{T} 1-\mathrm{T} 2$ & $10(21.3)$ \\
\hline T3-T4 & $37(78.7)$ \\
\hline \multicolumn{2}{|l|}{ Lymph node metastasis ${ }^{1}$} \\
\hline Positive & 17 (36.9) \\
\hline Negative & $29(63.1)$ \\
\hline \multicolumn{2}{|l|}{ Differentiation $^{2}$} \\
\hline G1 & $2(4.6)$ \\
\hline $\mathrm{G} 2$ & $39(88.6)$ \\
\hline G3 & $3(6.8)$ \\
\hline \multicolumn{2}{|l|}{ Clinical stage } \\
\hline I-II & 15 (31.9) \\
\hline III-IV & $32(68.1)$ \\
\hline \multicolumn{2}{|l|}{ Localization } \\
\hline Suprglottic & $5(10.6)$ \\
\hline Glottic & $5(10.6)$ \\
\hline Suprglottic and glottic & 7 (14.9) \\
\hline Subglottic & 0 \\
\hline Glottic and subglottic & $2(4.3)$ \\
\hline Hypopharynx & $3(6.4)$ \\
\hline Transglottic & $25(53.2)$ \\
\hline
\end{tabular}

${ }^{1}$ Data concerning lymph node status missing in 1 sample; ${ }^{2}$ Data concerning differentiation status missing in 3 samples.

their informed consent for inclusion before they participated in the study. The study was conducted in accordance with the Declaration of Helsinki, and the protocol was approved by the Ethics Committee of the Medical University of Lodz (RNN/222/13/KE 16 July 2013). The clinicopathological characteristic in laryngeal cancer patients are presented in Table 1.

\subsection{Total RNA isolation}

Total RNA was extracted from frozen tumour and non-tumour tissue with mirVana ${ }^{\mathrm{TM}}$ miRNA Isolation Kit (Ambion, Carlsbad, CA, USA) according to the manufacturer's instructions. RNA quality and purity was qualified by $260 / 280 \mathrm{~nm}$ absorbance measurement using Picodrop. Extracted RNA samples were stored at $-80^{\circ} \mathrm{C}$.

\subsection{Microarray experiment}

$500 \mathrm{ng}$ of total RNA from 3 patients (tumour and non-tumour tissue) was used for miRNA expression analysis. The small group of patients was examined to choose miRNAs for further investigation. Microarray 
Table 2

Primer sequences of tested genes

\begin{tabular}{llll}
\hline Gene & Forward primer 5'-3' & Reverse primer 5'-3' & Product length \\
\hline GAPDH & AGCCACATCGCTCAGACA & GCCCAATACGACCAAATCC & $66 \mathrm{bp}$ \\
PI3KR1 & CTCGGCCTCTTCCTGTTG & GCAAACTGCTCTGCAAGAT & $94 \mathrm{bp}$ \\
CXCR4 & CTCATCCTGGCCTTCATCA & ATCCAGACGCCAACATAGAC & $111 \mathrm{bp}$ \\
FZD4 & GTGCCCTTACCTCACAAAACC & ATCATAGCCACACTTGAGCAC & $123 \mathrm{bp}$ \\
HACE1 & AGAATGTTTTGTCACGTCTTTCG & AGTGCCAAAGCAATTCCAGAG & $124 \mathrm{bp}$ \\
CRK & ATGGCGGGCAACTTCGACT & AGTCCCGCACCAGGAACAC & $118 \mathrm{bp}$ \\
\hline
\end{tabular}

Table 3

Sequences of tested miRNA

\begin{tabular}{ll}
\hline miRNA & Sequence 3'-5' \\
\hline miR-21-3p & UGUCGGGUAGCUGACCACAAC \\
miR-139-3p & UGAGGUUGUCCCGGCGCAGAGG \\
miR-377-5p & CUUAAGUGGUCCCGUUGGAGA \\
$\operatorname{miR}-525-5 \mathrm{p}$ & UCUUUCACGUAGGGAGACCUC \\
$\operatorname{miR}-885-5 \mathrm{p}$ & UCUCCGUCCCAUCACAUUACCU \\
\hline
\end{tabular}

experiment was performed using Megaplex ${ }^{\mathrm{TM}}$ Pools Kit (Applied Biosystems, Foster City, CA, USA) according to the instructions. 384-well plates were run on a 7900HT Fast Real-Time PCR System (Applied Biosystems). The $2^{-\Delta \Delta C t}$ method was used to calculate relative expression.

\subsection{Reverse transcription}

cDNA synthesis of miRNA was performed using a TaqMan ${ }^{\circledR}$ MicroRNA Reverse Transcription Kit (Applied Biosystems, Foster City, CA, USA) according to the instructions. miRNA-specific RT primers and $10 \mathrm{ng}$ of total RNA was used to the reaction. The final volume of $15 \mu \mathrm{l}$ was incubated $\left(30 \mathrm{~min} 16^{\circ} \mathrm{C}, 30 \mathrm{~min} 42^{\circ} \mathrm{C}\right.$, $5 \mathrm{~min} 85^{\circ} \mathrm{C}$ ) in a thermocycler (Biometra). Following this, $400 \mathrm{ng}$ of the total RNA sample was added to the reaction tube to obtain specific mRNA cDNA. The cDNA was synthesised by priming with random hexamer primers, using the Improm II ${ }^{\mathrm{TM}}$ Reverse Transcription System (Promega, Madison, WI, USA), according to the manufacturer's protocol. The reaction tube containing the primer-RNA mix was incubated for $5 \mathrm{~min}$ at $70^{\circ} \mathrm{C}$ and chilled on ice. Next, the reverse transcription reaction mix was added to make a final volume of $100 \mu \mathrm{l}$. Samples were incubated for $5 \mathrm{~min}$ at $25^{\circ} \mathrm{C}, 60 \mathrm{~min}$ at $42^{\circ} \mathrm{C}$ and $15 \mathrm{~min}$ at $70^{\circ} \mathrm{C}$.

\subsection{Quantitative RT PCR}

To assess the level of miRNA expression, a TaqMan MicroRNA Assay (Applied Biosystems, Foster City, CA, USA) was used together with the TaqMan ${ }^{\circledR}$ Universal PCR Master Mix (Applied Biosystems, Foster City, CA, USA). Real-time PCR was performed using a Stratagene Mx3005P qPCR system. Each reaction contained $1 \mathrm{ng}$ of cDNA. The final volume was $20 \mu \mathrm{l}$. Samples were incubated for $10 \mathrm{~min}$ at $95^{\circ} \mathrm{C}$ followed by 40 cycles of $15 \mathrm{~s}$ at $95^{\circ} \mathrm{C}, 1 \mathrm{~min}$ at $60^{\circ} \mathrm{C}$, and run in triplicate. RNU 48 was used as an endogenous control to estimate the expression of miRNA genes. Comparative $\mathrm{Ct}$ method was chosen to determine relative quantification of miRNA. Brilliant II SYBR ${ }^{\circledR}$ Green QPCR Master Mix (Stratagene, La Jolla, CA, USA) was used to detect mRNA expression of five chosen genes. The reaction was performed with a Stratagene Mx3005P qPCR system. According to the manufacturer's instructions, of $10 \mathrm{ng}$ of cDNA and $100 \mathrm{nM}$ upstream and downstream primer were added per reaction. All samples were tested in $25 \mu \mathrm{l}$ reaction and performed in triplicate. Samples were incubated for $10 \mathrm{~min}$ at $95^{\circ} \mathrm{C}$ followed by 40 cycles of $30 \mathrm{~s}$ at $95^{\circ} \mathrm{C}$, $20 \mathrm{~s}$ at $60^{\circ} \mathrm{C}$. GAPDH was used an internal control. The $2^{-\Delta C t}$ value was calculated by subtracting the $\mathrm{Ct}$ of tested mRNA/miRNA from the Ct of GAPDH/RNU 48. The $2^{-\Delta \Delta C t}$ method was used to calculate relative expression. The primer sequences of the tested genes are presented in Table 2. The sequences of tested miRNA are presented in Table 3.

\subsection{Statistical analysis}

The results were ln-transformed to obtain a normal distribution and controlled for normal distribution using Shapiro-Wilk test. According to the results, non-parametric tests were used for non-normally distributed data, while parametric tests were used for normally distributed data. To assess the homogenity of variances, Levene's test was used. The difference between tumour and adjacent non-tumour tissue was assessed using the Wilcoxon signed rank test. The unpaired t-test was used to evaluate the relationship between miRNA and mRNA expression and clinicopathological parameters. To compare the differences between smoking duration groups, a one-way ANOVA was used. Correlations between the expression of miRNA and mRNA was identified by Pearson's 


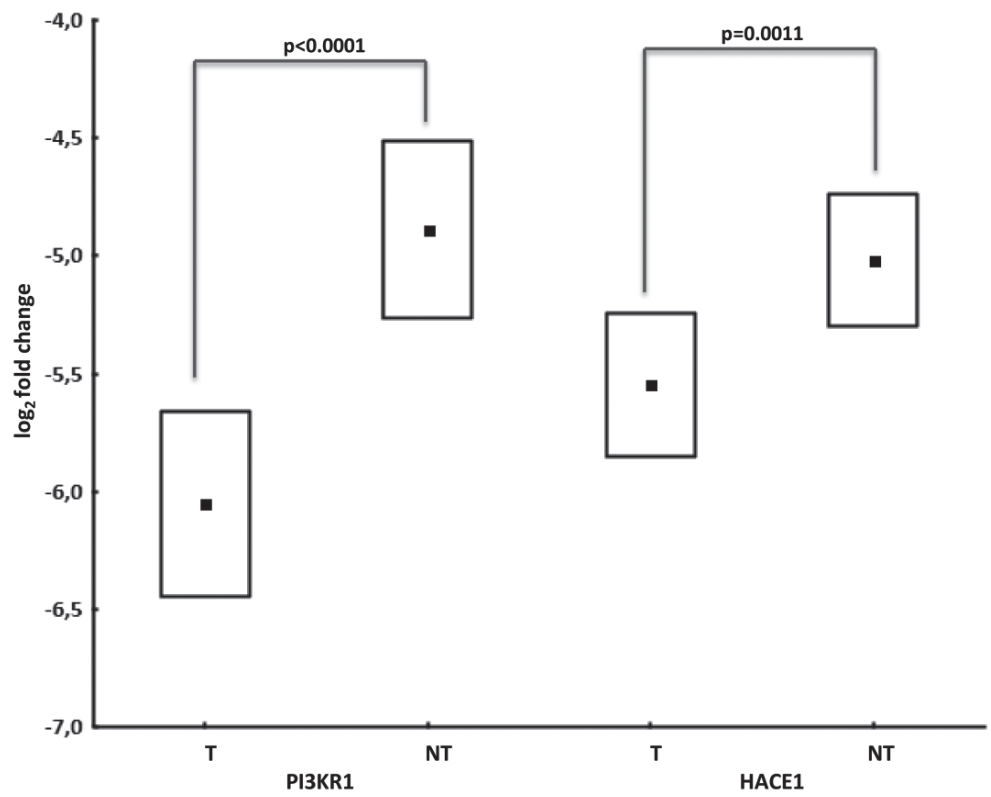

Fig. 1. Two genes with statistically significant expression (data presented as mean $\pm \mathrm{SE}$, (T) tumour, (NT) non-tumour).

correlation coefficient analysis. To contrast the differences between the absolute values of correlation coefficients between mRNA-mRNA, miRNA-miRNA and mRNA-miRNA, Welch's test was used. Discriminant analysis was conducted to find genes which can differentiate tumour tissue between normal tissue. Statistical analysis was performed using STATISTICA StatSoft Version 12. $P<0.05$ was considered statistically significant.

\section{Results}

\subsection{Differential levels of miRNA and mRNA in normal and tumour tissue}

To determine the expression of mRNA and miRNA in normal mucosa and tumour tissue, QPCR analysis was used. The data showed that all examined mRNAs were expressed less frequently in tumour tissue relative to normal samples. Among these analysed $\mathrm{mR}$ NAs, two genes, PIK3R1 and HACE1 were significantly down-regulated $(p<0.0001$ and $p=0.0011$ respectively) (Fig. 1). The average expression of PIK3RI $( \pm \mathrm{SE})$ in tumour and normal tissue was -6.05 ( \pm $0.39)$ and $-4.89( \pm 0.37)$ respectively. The average expression of HACE1 $( \pm \mathrm{SE})$ in tumour tissue was -5.55 $( \pm 0.31)$ as well as $-5.02( \pm 0.28)$ in adjacent normal mucosa. Moreover, the expression level of these genes were lower in both normal and tumour tissue
Table 4

The expression of miRNA and mRNA in tumour and non-tumour tissue determined by QPCR (data presented as mean $\pm \mathrm{SE}$ )

\begin{tabular}{lccr}
\hline gene & TUMOUR & NON-TUMOUR & $\mathrm{p}$ \\
\hline PIK3R1 & $-6.05 \pm 0.39$ & $-4.89 \pm 0.37$ & $<0.0001$ \\
CXCR4 & $-0.77 \pm 0.37$ & $-0.46 \pm 0.39$ & 0.2899 \\
FZD4 & $-1.27 \pm 0.41$ & $-0.81 \pm 0.41$ & 0.1561 \\
HACE1 & $-5.55 \pm 0.31$ & $-5.02 \pm 0.28$ & 0.0011 \\
CRK & $-0.98 \pm 0.39$ & $-0.79 \pm 0.37$ & 0.5049 \\
miR-21-3p & $-3.80 \pm 0.28$ & $-4.65 \pm 0.30$ & 0.0014 \\
miR-139-3p & $-7.35 \pm 0.21$ & $-6.41 \pm 0.19$ & 0.0013 \\
miR-377-5p & $-9.00 \pm 0.34$ & $-8.83 \pm 0.46$ & 0.3043 \\
miR-525-5p & $-9.06 \pm 0.48$ & $-10.66 \pm 0.57$ & 0.0063 \\
miR-885-5p & $-6.23 \pm 0.34$ & $-5.01 \pm 0.28$ & 0.0009 \\
\hline
\end{tabular}

compared to the expression level of $C X C R 4, F Z D 4$ and CRK genes.

Next, the data indicate that two types of miRNA, miR-21-3p and miR-525-5p, were expressed at a higher level in the tumour than in the corresponding adjacent normal tissue, with a significance of $p=$ 0.0014 (miR-21-3p) and $p=0.0063$ (miR-525-5p). The average expression $( \pm \mathrm{SE})$ in tumour and normal tissue was $-3.80( \pm 0.28)$ and $-4.65( \pm 0.30)$ respectively for miR-21-3p as well as $-9.06( \pm 0.48)$ and $-10.66( \pm 0.57)$ respectively for miR-525-5p. The next three miRNA were expressed less frequently in tumour samples, and significant down-regulation was observed for miR-139-3p ( $p=0.0013)$ and miR-885$5 \mathrm{p}(p=0.0009)$ (Fig. 2). The average expression $( \pm$ $\mathrm{SE})$ of miR-139-3p in tumour was $-7.35( \pm 0.21)$ and in normal mucosa $-6.41( \pm 0.19)$. The average ex- 


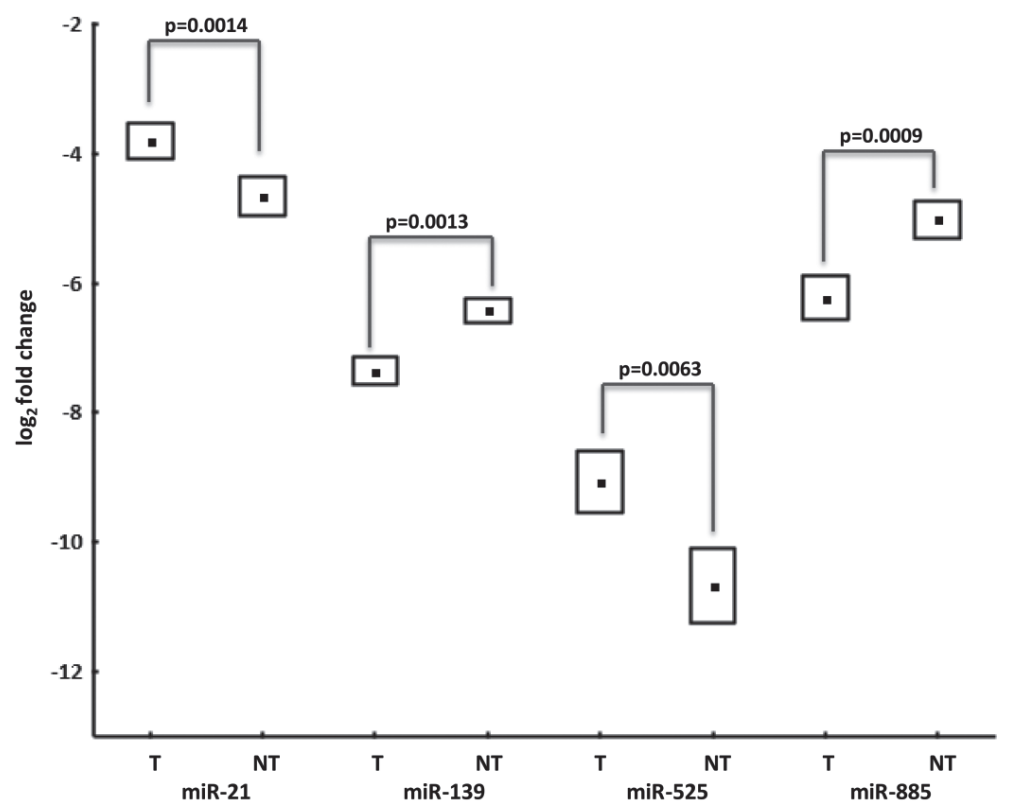

Fig. 2. Four miRNA with statistically significant expression (data presented as mean $\pm \mathrm{SE}$, (T) tumour, (NT) non-tumour).

pression $( \pm \mathrm{SE}$ ) of miR-885-5p in tumour and adjacent normal tissue was $-6.23( \pm 0.34)$ and $-5.01( \pm 0.28)$ respectively. The data are presented in Table 4.

\subsection{Association between expression level of miRNA and $m R N A$ in different clinicopathological characteristics}

No association was observed between sex, age, $\mathrm{T}$ classification, lymph node metastasis, clinical stage and smoking duration.

\subsection{Correlation between the expression of miRNAs, mRNAs and miRNA to mRNA}

The results reveal a correlation between the relative expression of different miRNA. The strongest positive correlation was observed between miR-377 and miR$525(r=0.59, p=0.003)$. The levels of expression of miR-21 and miR-139, as well as of miR-525 and miR-885, were found to be medium correlated $(r=$ 0.35). TargetScan, DIANA Lab and miRanda software also indicate a correlation between miRNA which possess the same putative target gene (Table 5). miR-525 was positively correlated to miR-885 $(r=0.35, p=$ $0.04)$ and miR-21 ( $r=0.41, p=0.015)$. Next, among the tested genes, the strongest positive correlation was demonstrated by $C R K$ vs $C X C R 4(r=0.92, p<0.01)$ and $C R K$ vs FZD4 ( $r=0.91, p<0.001)$. The relative expression level of $P I K 3 R I$ was medium correlated to all four genes. However, no correlation was observed between miRNAs and mRNAs. Moreover, the results of Welch's test indicate that the correlation between miRNAs and mRNAs was higher than that of miRNA vs mRNA. The absolute value of the mean correlation coefficient $( \pm$ SE) was $0.14( \pm 0.01)$ for miRNA vs mRNA and $0.41( \pm 0.06)$ for miRNAs and mRNAs $(p=0.0002)$. The data are presented in Tables 6 and 7.

\subsection{Combinations of genes which can differentiate tumour tissue from non-tumour tissue}

Discriminant analysis was conducted to determine which of the tested genes or miRNA can distinguish between tumour tissue and normal tissue. To obtain the normal distribution, the data was transformed using a Box-Cox transformation (miR-885 $\lambda=-0.111$ and PIK3R1 $\lambda=-0.117)$. The forward stepwise mode revealed that miR-885 and $P I K 3 R I$ correctly differentiated tumour and non-tumour tissue with a median accuracy of $72 \%$ (Wilk's lambda $=0.8, p<0.0001$ ). The average expression of miR-885 $( \pm \mathrm{SE})$ in tumour tissue was $-6.23( \pm 0.34)$ as well as $-5.01( \pm 0.28)$ in adjacent normal mucosa. The average expression of PIK3R1 ( $\pm \mathrm{SE}$ ) in tumour and normal tissue was $-6.05( \pm 0.39)$ and $-4.89( \pm 0.37)$ respectively. 
Table 5

miRNA target prediction (underline letters indicate binding pattern with their predicted targets)

\begin{tabular}{lllll}
\hline miRNA & Sequence 3'-5' & Target gene & 3'UTR position & Seed match \\
\hline miR-21-3p & UGUCGGGUAGCUGACCACAAC & FZD4 & $574-579$ & $8 \mathrm{mer}$ \\
miR-525-5p & UCUUUCACGUAGGGAGACCUC & PIK3R1 & $48-54$ & $8 \mathrm{mer}$ \\
& UCUUUCACGUAGGGAGACCUC & FZD4 & $955-961$ & $7 \mathrm{mer} 1 \mathrm{~A}$ \\
& UCUUUCACGUAGGG $\overline{\text { AGACCUCC }}$ & HACE1 & $6-12$ & $7 \mathrm{mer} 1 \mathrm{~A}$ \\
miR-885-5p & UCUCCGUCCCAUCACAUUACCU & HACE1 & $572-578$ & $8 \mathrm{mer}$ \\
\hline
\end{tabular}

Table 6

Correlation between relative expression level of miRNAs ( $\mathrm{r}$ - Pearson's correlation coefficient)

\begin{tabular}{llllll}
\hline miRNA & miR-21 & miR-139 & miR-377 & miR-525 & miR-885 \\
\hline miR-21 & $r=1.00$ & $r=0.35$ & $r=-0.02$ & $r=0.41$ & $r=0.49$ \\
& & $p=0.02$ & $p=0.90$ & $p=0.015$ & $p<0.001$ \\
miR-139 & $r=035$ & $r=1.00$ & $r=0.30$ & $r=0.13$ & $r=0.48$ \\
& $p=0.02$ & & $p=0.12$ & $p=0.48$ & $p=0.001$ \\
miR-377 & $r=-0.02$ & $r=0.30$ & $r=1.00$ & $r=0.59$ & $r=0.13$ \\
& $p=0.90$ & $p=0.12$ & & $p=0.003$ & $p=0.48$ \\
miR-525 & $r=0.41$ & $r=0.13$ & $r=0.59$ & $r=1.00$ & $r=0.35$ \\
& $p=0.015$ & $p=0.48$ & $p=0.003$ & & $p=0.04$ \\
miR-885 & $r=0.49$ & $r=0.49$ & $r=0.13$ & $r=0.35$ & $r=1.00$ \\
& $p<0.001$ & $p=0.001$ & $p=0.48$ & $p=0.04$ & \\
\hline
\end{tabular}

Table 7

Correlation between relative expression of mRNAs ( $\mathrm{r}$ - Pearson's correlation coefficient)

\begin{tabular}{llllll}
\hline gene & PIK3R1 & CXCR4 & FZD4 & HACE1 & CRK \\
\hline PIK3R1 & $r=1.00$ & $r=0.41$ & $r=0.41$ & $r=0.53$ & $r=0.41$ \\
& & $p=0.005$ & $p=0.005$ & $p>0.001$ & $p=0.006$ \\
CXCR4 & $r=0.41$ & $r=1.00$ & $r=0.93$ & $r=0.08$ & $r=0.92$ \\
& $p=0.005$ & & $p<0.01$ & $p=0.57$ & $p<0.01$ \\
FZD4 & $r=0.41$ & $r=0.93$ & $r=1.00$ & $r=0.21$ & $r=0.91$ \\
& $p=0.005$ & $p<0.01$ & & $p=0.15$ & $p<0.001$ \\
HACE1 & $r=0.53$ & $r=0.08$ & $r=0.21$ & $r=1.00$ & $r=0.14$ \\
& $p<0.001$ & $p=0.57$ & $p=0.15$ & & $p=0.33$ \\
CRK & $r=0.41$ & $r=0.92$ & $r=0.91$ & $r=0.14$ & $r=1.00$ \\
& $p=0.006$ & $p<0.01$ & $p<0.001$ & $p=0.33$ & \\
\hline
\end{tabular}

\section{Discussion}

The present study investigates five miRNA and their relevance to laryngeal squamous cell carcinoma. One of the most frequently examined miRNAs in human neoplasm is miR-21 [12]. Although many studies have indicated that miR-21 is overexpressed in a variety of malignancies, including LSCC [33], they have examined miR-21-5p. The present study analyzes miR-21$3 \mathrm{p}\left(\mathrm{miR}-21^{*}\right)$, which is known to also be up-regulated in cancer tissue, as notated by Lu et al. [20]. miR-21-3p overexpression has also been determined elsewhere in a group of head and neck cancers [8]. While the sample size in present study may have been too small to determine any other correlations, other studies have also failed to describe any association between the expression profile of miR-21-5p and clinicopathological parameters in LSCC [12]. Nevertheless, the present study is the first to investigate the relationship miR-21-3p ex- pression and these features in LSCC. Our findings suggest that the miRNA passenger strand, poorly investigated in cancer, may play an important role in laryngeal cancer. It will be worth to examine both strands in this type of malignancy to determine if there is any connection in their action.

One of the most intensively investigated signalling pathways in the remarkable spectrum of tumour types is the PI3 kinase pathway. PI3 kinase IA is a heterodimer that consists of a catalytic (p110 $\alpha)$ and a regulatory subunit $(\mathrm{p} 85 \alpha)$. Loss of the $\mathrm{p} 85 \alpha$ subunit leads to increased kinase activity. The regulatory subunit is encoded by $P I K 3 R 1$, which is known to be mutated in various malignancies [14]. Interestingly, Lui et al. report that $\mathrm{PI} 3 \mathrm{~K}$ pathway mutations were more frequent in laryngeal cancer than in other HNSCC [21]. The present study indicates that PIK3RI expression is significantly down-regulated in LSCC samples, as confirmed by previous studies concerning breast can- 
cer [2]. Possibly, PIK3RI underexpression leads to the activation of downstream PI3K signalling and promotes the development of laryngeal cancer. In HNSCC mutations of PIK3R1 were also found what may suggest the same mechanism of down-regulation like in studies mentioned above concerning breast cancer [2]. However, there are almost no reports about regulatory subunit's mutations exactly in laryngeal cancer. Thus, additional studies are needed to reveal their role in this type of tumour. Another plausible mechanism of altered expression may be miRNA's regulation. Furthermore, based on the miRNA prediction target algorithms used in the present study, $P I K 3 R I$ was indicated as a potential target of miR-525-5p, which was found to be overexpressed in laryngeal cancer samples compares to normal tissue. However, as no correlation was found between the expression level of the tested miRNAs and mRNAs, experimental validation is required.

Much fewer studies have been conducted on miR525 in human malignancies. While a few reports do concern the overexpression of miR-525-3p and its role in liver cancer cell migration and invasion [29], none investigate this type of miRNA in LSCC or in head and neck cancers. The gene encodes miR-525 is reported to be located in a copy number amplified region $19 q 13.42$. In HNSCC there is no reports about this alteration, however, many authors present only frequent aberrations. It may suggest that this alteration is rare. According to miRNA prediction target algorithms both miR-525-5p and miR-21-3p have the same potential target gene, FZD4, and are positively correlated, which may suggest their synergistic action in mRNA silencing. However, no association was found.

FZD4 encodes the frizzled 4 receptor, a member of the GPCR family of receptors and a principal receptor of the Wnt signalling pathway - implicated in various cancer types including colon and prostate [28], though little data is available for HNSCC. The Wnt pathway is involved in epithelial-to-mesenchymal transition (EMT) and development of metastasis [43]. Our findings are in contrary to those of some studies which determine FZD4 overexpression. But these studies address other cancer types: prostate [10] and acute myeloid leukemia [39]. Generally, little is known about the role of frizzled receptors in human malignancies and there only a few studies have been published concerning this class of receptor in HNSCC [4]. The Wnt signalling pathway is poorly understood in laryngeal cancer. Moreover, Galera-Ruiz et al. suggest that the canonical Wnt pathway may be inactive in LSCC [7], though Psyrri et al. determined its prognostic value in this form of cancer [31]. As knowledge of the noncanonical Wnt pathway and its role in cancer is limited, further studies are required.

Another poorly investigated miRNA in human cancers is miR-377-5p (miR-377*) compared to miR-377$3 p$. While no differences in miR-377-5p expression were observed between tumour and normal samples, nor any association with clinicopathological parameters. What is more, miR-377-5p level is correlated with miR-525-5p, indicating that these miRNA may bind to the same 3'UTR region of the target gene which were not investigated in this study. Interestingly, in $23 \%$ of examined tumour samples the expression of miR-377$5 \mathrm{p}$ was extremely low. The gene encodes $m i R-377$ is located in a region $14 \mathrm{q} 32.31$ which was found to be lost or epigenetically silenced in prostate cancer [6]. On the other hand in laryngeal cancer 14q24-32 area was identified as copy number region, however, this alteration is rare. Possibly, in some cases of LSCC microdeletions of this region are presented which have never been detected before. Thus, further studies with larger sample sizes are needed to establish the role of this miRNA in laryngeal cancer.

miR-139-3p was also found to be significantly down-regulated in laryngeal cancer tissue. Our results are consistent with Sun et al., who also reveal differential miR-139-3p expression between positive and negative lymph node metastasis tumours [37]. The current study reveals no association between the expression of miR-139-3p and lymph node status, perhaps because the LSCC samples were more heterogenous and the aim of abovementioned study was supraglottic LSCC. miR-139-3p was also found to be underexpressed in colorectal cancer [35]. Interestingly, Lin et al. [19] note miR-139-3p up-regulation in CRC with liver metastasis, which may indicate that this miRNA plays a variable role as both oncogene and suppressor in different tumours. Similarly, the frequently studied miR-139-5p also appears to act as oncogene and suppressor gene and regulate multiple processes in various malignancies [44]. Moreover, miR-139-3p is positively correlated with miR-21-3p and miR-885-5p. Possibly, these miRNAs have the same target gene that was not examined in the present study.

Another poorly-understood issue related to cancer is the role of chemokine pathways. These small molecules are known to have a multi-faceted and very complex involvement in cancer. Many studies have reported that various malignancies, including those of the lung and kidney, use chemokine pathways to disseminate cancer cells to target organs [41]. Sev- 
eral studies have indicated that the CXCR4/CXCL12 axis also plays a crucial role in promoting metastasis in laryngeal cancer [38]. CXCR4 encodes C$\mathrm{X}-\mathrm{C}$ chemokine receptor 4 , a member of GPCR receptor family which is found to be overexpressed in many cancer types such as HNSCC. While Luo et al. found CXCR4 overexpression to be associated with metastatic LSCC [22], no differences in CXCR4 expression were observed between cancer tissue and counterpart normal tissue in the present study, and no relationship was found with cancer lymph node metastasis. The reason for this discrepancy may be that while both Tan et al. [38] and Luo et al. [22] found their group of laryngeal cancer samples to contain more lymph node positive samples, $63 \%$ of the samples in our group were lymph node negative. CXCR4 overexpression could potentially act as an indicator of lymph node metastasis, but additional studies are needed. Furthermore, CXCR4 mRNA was positively correlated with $P I K 3 R 1$, indicating that $C X C R 4$ is co-expressed with one of the elements of its pathway.

To date, there are only several studies concerning miR-885 in human cancer have been published. Afanasyeva et al. demonstrate miR-885-5p underexpression in neuroblastoma and its role in inhibiting cancer cell proliferation, survival and apoptosis promotion [1]. Conversely, Hur et al. determined that miR885-5p is up-regulated in CRC with liver metastasis, however, they found no relationship between clinicopathological characteristics. Still, high serum miR885-5p expression was associated with lymph node metastasis, distant metastasis and poor survival [13]. The present study is the first to verify the underexpression of miR-885-5p in LSCC tissue. One of the most frequently deleted area in laryngel cancer is chromosom $3 p$ [24]. The gene encodes miR-885 is located at 3 p25.3 region, which may suggest the reason of miRNA's downregulation in this type of tumour. All studies demonstrate the variable character of miR-885$5 \mathrm{p}$ in tumours originating from different tissue. More studies will be needed to define the role of miR-885$5 \mathrm{p}$ in laryngeal cancer. Our findings also suggest that miR-885-5p and PIK3RI are the best indicators for the classification of laryngeal cancer tissue and normal mucosa. However, while it is tempting to speculate that PIK3Rl and miR-885-5p might be good LSCC biomarkers and could be used to examine the margin after surgery, additional studies are required. Furthermore, both miR-885-5p and miR-525-5p bind to the predicted target gene HACE1 and are positively correlated, which may indicate their synergistic action in mRNA silencing. However, no association was revealed, and therefore, experimental analyses are again needed to validate these findings.

HACE1 encodes E3 ubiquitin ligase, a HECT family ubiquitin ligase that possesses the unique capability of intrinsic catalytic activity. HECT E3 ligase targets the Rac1 gene for subsequent proteosomal degradation and regulates cell motility and apoptosis [17]. Downregulation of HACE1 due to allelic loss or promoter hypermethylation, was reported in multiple human cancers such as gastric [34] and breast cancer [9], leading to enhanced Rac1 signalling and promoting cell invasion. Similarly, although our findings indicate HACE1 underexpression in laryngeal cancer tissue, the present study does not examine the mechanism of this alteration, and as a literature search reveals no other studies concerning the role of HECT E3 ubiquitin ligase in this type of cancer, further studies are needed to establish other HACE1 targets.

Unanswered questions remain about the role of CRK adaptor proteins in tumorgenesis. CRK is involved in signal transduction through protein-protein interactions. CRK mediates signal propagation of multiple cancer-related processes, such as cell proliferation and migration [18]. In several malignancies for instance lung cancer, CRK was found to be overexpressed [26]. The present study indicates that the expression of CRK mRNA remains unchanged between laryngeal cancer tissue and normal mucosa, as well as between clinical stages. Although Miller et al. [26] also failed to find any differences in lung cancer, they did find higher $C R K$ expression at more advanced clinical stages. CRK is known to interact with the p85 $\alpha$ subunit and lead to PI3K signaling pathway activation [25], which may account for the strong positive correlation observed in the present study between $C R K$ mRNA and FZD4 or CXCR4, and the medium correlation with $P I K 3 R 1$. What is more, CRK mediates small G- protein interactions, and both CXCR4 and FZD4 are members of the GPCR family [5].

In conclusion, the change in miRNA expression is frequent than the expression of other tested genes. Moreover, the expression of passenger strands such as miR-21-3p and miR-139-3p, which are rarely investigated in tumors, is also significantly affected in laryngeal cancer and may play an important role in this type of malignancy. It will be worth to examine both strands in this type of tumour to determine if there is any connection in their action. While PIK3R1, HACE1, miR139-3p, and miR-885-5p may act as tumor suppressor genes in the studied tumour type, miR-21-3p and miR- 
525-5p seem to have oncogenic properties. Our findings suggest that miR-885-5p and PIK3RI are the best indicators for the classification of laryngeal cancer tissue and normal mucosa. Our study contributes further to the body of evidence on which further investigations concerning laryngeal cancer can be based. Additional studies are needed to determine the functions of miRNAs and genes analyzed in this report as well as the clinical significance of these findings.

\section{Acknowledgments}

This research was supported by Medical University of Lodz grants no. 502-64-065 and 502-24-014.

\section{References}

[1] E.A. Afanasyeva, P. Mestdagh, C. Kumps, J. Vandesompele, V. Ehemann, J. Theissen, M. Fischer, M. Zapatka, B. Brors, L. Savelyeva, V. Sagulenko, F. Speleman, M. Schwab and F. Westermann, MicroRNA miR-885-5p targets CDK2 and MCM5, activates p53 and inhibits proliferation and survival, Cell Death Differ 18 (2011), 974-84.

[2] M. Cizkova, S. Vacher, D. Meseure, M. Trassard, A. Susini, D. Mlcuchova, C. Callens, E. Rouleau, F. Spyratos, R. Lidereau and I. Bieche, PIK3R1 underexpression is an independent prognostic marker in breast cancer, BMC Cancer 13 (2013), 545.

[3] E. Coskunpinar, Y.M. Oltulu, K.S. Orhan, N.O. Tiryakioglu, D. Kanliada and F. Akbas, Identification of a differential expression signature associated with tumorigenesis and metastasis of laryngeal carcinoma, Gene 534 (2014), 183-8.

[4] S.M. Diaz Prado, V. Medina Villaamil, G. Aparicio Gallego, M. Blanco Calvo, J.L. Lopez Cedrun, S. Sironvalle Soliva, M. Valladares Ayerbes, R. Garcia Campelo and L.M. Anton Aparicio, Expression of Wnt gene family and frizzled receptors in head and neck squamous cell carcinomas, Virchows Arch 455 (2009), 67-75.

[5] S.M. Feller, Crk family adaptors-signalling complex formation and biological roles, Oncogene 20 (2001), 6348-71.

[6] A. Formosa, E.K. Markert, A.M. Lena, D. Italiano, E. FinazziAgro, A.J. Levine, S. Bernardini, A.V. Garabadgiu, G. Melino and E. Candi, MicroRNAs, miR-154, miR-299-5p, miR-376a, miR-376c, miR-377, miR-381, miR-487b, miR-485-3p, miR495 and miR-654-3p, mapped to the $14 q 32.31$ locus, regulate proliferation, apoptosis, migration and invasion in metastatic prostate cancer cells, Oncogene 33 (2014), 5173-82.

[7] H. Galera-Ruiz, M.J. Rios-Moreno, R. Gonzalez-Campora, I. Ortega, A. Fernandez, A. Garcia-Escudero and H. GaleraDavidson, The cadherin-catenin complex in laryngeal squamous cell carcinoma, Eur Arch Otorhinolaryngol 269 (2012), 1183-8.

[8] F. Ganci, A. Sacconi, N. Bossel Ben-Moshe, V. Manciocco, I. Sperduti, L. Strigari, R. Covello, M. Benevolo, E. Pescarmona, E. Domany, P. Muti, S. Strano, G. Spriano, G. Fontemaggi and G. Blandino, Expression of TP53 mutationassociated microRNAs predicts clinical outcome in head and neck squamous cell carcinoma patients, Ann Oncol 24 (2013), 3082-8.
[9] E.T. Goka and M.E. Lippman, Loss of the E3 ubiquitin ligase HACE1 results in enhanced Rac1 signaling contributing to breast cancer progression, Oncogene (2015).

[10] S. Gupta, K. Iljin, H. Sara, J.P. Mpindi, T. Mirtti, P. Vainio, J. Rantala, K. Alanen, M. Nees and O. Kallioniemi, FZD4 as a mediator of ERG oncogene-induced WNT signaling and epithelial-to-mesenchymal transition in human prostate cancer cells, Cancer Res 70 (2010), 6735-45.

[11] H.M. Heneghan, N. Miller and M.J. Kerin, MiRNAs as biomarkers and therapeutic targets in cancer, Curr Opin Pharmacol 10 (2010), 543-50.

[12] A. Hu, J.J. Huang, W.H. Xu, X.J. Jin, J.P. Li, Y.J. Tang, X.F. Huang, H.J. Cui and G.B. Sun, miR-21 and miR-375 microRNAs as candidate diagnostic biomarkers in squamous cell carcinoma of the larynx: association with patient survival, Am J Transl Res 6 (2014), 604-13.

[13] K. Hur, Y. Toiyama, A.J. Schetter, Y. Okugawa, C.C. Harris, C.R. Boland and A. Goel, Identification of a metastasisspecific MicroRNA signature in human colorectal cancer, $J$ Natl Cancer Inst 107 (2015).

[14] B.S. Jaiswal, V. Janakiraman, N.M. Kljavin, S. Chaudhuri, H.M. Stern, W. Wang, Z. Kan, H.A. Dbouk, B.A. Peters, P. Waring, T. Dela Vega, D.M. Kenski, K.K. Bowman, M. Lorenzo, H. Li, J. Wu, Z. Modrusan, J. Stinson, M. Eby, P. Yue, J.S. Kaminker, F.J. de Sauvage, J.M. Backer and S. Seshagiri, Somatic mutations in p85alpha promote tumorigenesis through class IA PI3K activation, Cancer Cell 16 (2009), 463-74.

[15] F. Jenckel and R. Knecht, State of the art in the treatment of laryngeal cancer, Anticancer Res 33 (2013), 4701-10.

[16] A.W. Joseph and G. D'Souza, Epidemiology of human papillomavirus-related head and neck cancer, Otolaryngol Clin North Am 45 (2012), 739-64.

[17] C. Kucuk, X. Hu, J. Iqbal, P. Gaulard, D. Klinkebiel, A. Cornish, B.J. Dave and W.C. Chan, HACE1 is a tumor suppressor gene candidate in natural killer cell neoplasms, Am J Pathol 182 (2013), 49-55.

[18] S. Kumar, J.E. Fajardo, R.B. Birge and G. Sriram, Crk at the quarter century mark: perspectives in signaling and cancer, $J$ Cell Biochem 115 (2014), 819-25.

[19] M. Lin, W. Chen, J. Huang, H. Gao, Y. Ye, Z. Song and X. Shen, MicroRNA expression profiles in human colorectal cancers with liver metastases, Oncol Rep 25 (2011), 739-47.

[20] Z.M. Lu, Y.F. Lin, L. Jiang, L.S. Chen, X.N. Luo, X.H. Song, S.H. Chen and S.Y. Zhang, Micro-ribonucleic acid expression profiling and bioinformatic target gene analyses in laryngeal carcinoma, Onco Targets Ther 7 (2014), 525-33.

[21] V.W. Lui, M.L. Hedberg, H. Li, B.S. Vangara, K. Pendleton, Y. Zeng, Y. Lu, Q. Zhang, Y. Du, B.R. Gilbert, M. Freilino, S. Sauerwein, N.D. Peyser, D. Xiao, B. Diergaarde, L. Wang, S. Chiosea, R. Seethala, J.T. Johnson, S. Kim, U. Duvvuri, R.L. Ferris, M. Romkes, T. Nukui, P. Kwok-Shing Ng, L.A. Garraway, P.S. Hammerman, G.B. Mills and J.R. Grandis, Frequent mutation of the PI3K pathway in head and neck cancer defines predictive biomarkers, Cancer Discov 3 (2013), 7619.

[22] H.N. Luo, Z.H. Wang, Y. Sheng, Q. Zhang, J. Yan, J. Hou, K. Zhu, Y. Cheng, Y.L. Xu, X.H. Zhang, M. Xu and X.Y. Ren, MiR-139 targets CXCR4 and inhibits the proliferation and metastasis of laryngeal squamous carcinoma cells, Med Oncol 31 (2014), 789.

[23] J.P. Machiels, M. Lambrecht, F.X. Hanin, T. Duprez, V. Gregoire, S. Schmitz and M. Hamoir, Advances in the man- 
agement of squamous cell carcinoma of the head and neck, F1000Prime Rep 6 (2014), 44.

[24] A.A. Makitie and O. Monni, Molecular profiling of laryngeal cancer, Expert Rev Anticancer Ther 9 (2009), 1251-60.

[25] P. Mellor, L.A. Furber, J.N. Nyarko and D.H. Anderson, Multiple roles for the p85alpha isoform in the regulation and function of PI3K signalling and receptor trafficking, Biochem J 441 (2012), 23-37.

[26] C.T. Miller, G. Chen, T.G. Gharib, H. Wang, D.G. Thomas, D.E. Misek, T.J. Giordano, J. Yee, M.B. Orringer, S.M. Hanash and D.G. Beer, Increased C-CRK proto-oncogene expression is associated with an aggressive phenotype in lung adenocarcinomas, Oncogene 22 (2003), 7950-7.

[27] R.S. Ni, X. Shen, X. Qian, C. Yu, H. Wu and X. Gao, Detection of differentially expressed genes and association with clinicopathological features in laryngeal squamous cell carcinoma, Oncol Lett 4 (2012), 1354-1360.

[28] C. Niehrs, The complex world of WNT receptor signalling, Nat Rev Mol Cell Biol 13 (2012), 767-79.

[29] F. Pang, R. Zha, Y. Zhao, Q. Wang, D. Chen, Z. Zhang, T. Chen, M. Yao, J. Gu and X. He, MiR-525-3p enhances the migration and invasion of liver cancer cells by downregulating ZNF395, PLoS One 9 (2014), e90867.

[30] B. Perez-Ordonez, M. Beauchemin and R.C. Jordan, Molecular biology of squamous cell carcinoma of the head and neck, J Clin Pathol 59 (2006), 445-53.

[31] A. Psyrri, V. Kotoula, E. Fountzilas, Z. Alexopoulou, M. Bobos, D. Televantou, G. Karayannopoulou, D. Krikelis, K. Markou, I. Karasmanis, N. Angouridakis, K.T. Kalogeras, A. Nikolaou and G. Fountzilas, Prognostic significance of the Wnt pathway in squamous cell laryngeal cancer, Oral Oncol 50 (2014), 298-305.

[32] H. Ramroth, A. Schoeps, E. Rudolph, G. Dyckhoff, P. Plinkert, B. Lippert, K. Feist, K.W. Delank, K. Scheuermann, G. Baier, I. Ott, S. Chenouda, H. Becher and A. Dietz, Factors predicting survival after diagnosis of laryngeal cancer, Oral Oncol 47 (2011), 1154-8.

[33] J. Ren, D. Zhu, M. Liu, Y. Sun and L. Tian, Downregulation of miR-21 modulates Ras expression to promote apoptosis and suppress invasion of Laryngeal squamous cell carcinoma, Eur J Cancer 46 (2010), 3409-16.

[34] M. Sakata, Y.H. Kitamura, K. Sakuraba, T. Goto, H.
Mizukami, M. Saito, K. Ishibashi, G. Kigawa, H. Nemoto, Y. Sanada and K. Hibi, Methylation of HACE1 in gastric carcinoma, Anticancer Res 29 (2009), 2231-3.

[35] K. Shen, Q. Liang, K. Xu, D. Cui, L. Jiang, P. Yin, Y. Lu, Q. Li and J. Liu, MiR-139 inhibits invasion and metastasis of colorectal cancer by targeting the type I insulin-like growth factor receptor, Biochem Pharmacol 84 (2012), 320-30.

[36] O. Slaby, J. Bienertova-Vasku, M. Svoboda and R. Vyzula, Genetic polymorphisms and microRNAs: new direction in molecular epidemiology of solid cancer, J Cell Mol Med $\mathbf{1 6}$ (2012), 8-21.

[37] X. Sun, Y. Song, X. Tai, B. Liu and W. Ji, MicroRNA expression and its detection in human supraglottic laryngeal squamous cell carcinoma, Biomed Rep 1 (2013), 743-746.

[38] C.T. Tan, C.Y. Chu, Y.C. Lu, C.C. Chang, B.R. Lin, H.H. Wu, H.L. Liu, S.T. Cha, E. Prakash, J.Y. Ko and M.L. Kuo, CXCL12/CXCR4 promotes laryngeal and hypopharyngeal squamous cell carcinoma metastasis through MMP-13dependent invasion via the ERK1/2/AP-1 pathway, Carcinogenesis 29 (2008), 1519-27.

[39] L. Tickenbrock, S. Hehn, B. Sargin, C. Choudhary, N. Baumer, H. Buerger, B. Schulte, O. Muller, W.E. Berdel, C. Muller-Tidow and H. Serve, Activation of Wnt signalling in acute myeloid leukemia by induction of Frizzled-4, Int J Oncol 33 (2008), 1215-21.

[40] M.C. Torrente, J.P. Rodrigo, M. Haigentz, Jr., F.G. Dikkers, A. Rinaldo, R.P. Takes, J. Olofsson and A. Ferlito, Human papillomavirus infections in laryngeal cancer, Head Neck $\mathbf{3 3}$ (2011), 581-6.

[41] P. Weitzenfeld and A. Ben-Baruch, The chemokine system, and its CCR5 and CXCR4 receptors, as potential targets for personalized therapy in cancer, Cancer Lett 352 (2014), 3653.

[42] A. Wojcicka, A. de la Chapelle and K. Jazdzewski, MicroRNA-related sequence variations in human cancers, Hum Genet 133 (2014), 463-9.

[43] D. Yao, C. Dai and S. Peng, Mechanism of the mesenchymalepithelial transition and its relationship with metastatic tumor formation, Mol Cancer Res 9 (2011), 1608-20.

[44] H.D. Zhang, L.H. Jiang, D.W. Sun, J. Li and J.H. Tang, MiR-139-5p: promising biomarker for cancer, Tumour Biol 36 (2015), 1355-65. 